\title{
Single Photon Emission Computed Tomography Scan as a Diagnostic Tool in Children with Cerebral Palsy Treated with Human Embryonic Stem Cells
}

\author{
Geeta Shroff ${ }^{1}$, Barthakur $\mathrm{JK}^{2}$, Parul Mohan ${ }^{3}$ and Harsh Mahajan ${ }^{4}$ \\ ${ }^{1}$ Nutech Mediworld, New Delhi, India \\ ${ }^{2}$ Department of Statistics, Retired Additional Secretary, Ministry of Home Affairs, Government of India \\ ${ }^{3}$ Department of Nuclear Medicine, Mahajan Imaging Centre, Fortis Flt. Lt. Rajan Dhall Hospital, New Delhi, India \\ ${ }^{4}$ Mahajan Imaging Centre, New Delhi, India
}

*Corresponding author: Geeta Shroff, Director, Nutech Mediworld, H-8, Green Park Extension, New Delhi-110016, India, Tel: +91 11 26565548; Fax: 91 11 46067841; E-mail: geetashroff@hotmail.com

Received date: Mar 16, 2015, Accepted date: Apr 11, 2015, Publication date: Apr 16, 2015

Copyright: () 2015 Shroff G, et al. This is an open-access article distributed under the terms of the Creative Commons Attribution License, which permits unrestricted use, distribution, and reproduction in any medium, provided the original author and source are credited.

\begin{abstract}
Introduction: Cerebral palsy (CP) is caused by abnormalities in the developing brain leading to an impairment of muscle coordination and movement control in the body. Neonatal hypoxic-ischemic brain injury and hypoxic ischemic encephalopathy (HIE) are major causes of $\mathrm{CP}$. Neurodegenerative disorders can be treated with human embryonic stem cells (hESC). Magnetic resonance imaging (MRI), positron emission tomography and single photon emission computed tomography (SPECT) scan are some of the imaging modalities for CP, but among all SPECT scan is more sensitive in detecting abnormalities in the brain. SPECT scan is an effective tool for the detection of cerebral blood flow, cerebral impairment and local brain metabolism. The present study was conducted to evaluate the diagnostic modality of SPECT scan that can assess the patient with CP before and after hESC therapy.
\end{abstract}

Methodology: The study included the patients with CP for whom SPECT scan was performed before and after the therapy. Technetium-hexa methyl propylene aminoxime (Tc-HMPAO) SPECT scan was analyzed to assess the number of hypoperfused lesions/ areas in the brain and the percentage of improvement by hESC treatment. The patients were scored clinically using GMFCS scores.

Results: Overall, 88 patients aged 2 months to 18 years with clinical manifestations of CP had SPECT scan performed before and after the therapy. The total treatment days varied from 31 to 310 days. All the subjects showed improvement in SPECT scan. Majority of subjects $(n=58)$ had significant SPECT scan $(>60 \%)$ change, followed by moderate SPECT scan $(>30 \%<60 \%)$ change in 15 subjects, mild scan $(>10 \%<30 \%)$ change in 3 subjects. These patients also showed a clinical improvement as reflected in their GMFCS scores.

Conclusion: SPECT scan can be a crucial diagnostic modality to assess patients with CP.

Keywords: Cerebral palsy; Human embryonic stem cells; SPECT scan; GMFCS

\section{Introduction}

Cerebral palsy (CP) is a neurological disorder caused by abnormalities in the developing brain of infants and children. CP leads to an impairment of muscle coordination in the body and movement control [1]. The worldwide prevalence of CP is 1.5 to 4 per 1,000 live births [2].

Developmental brain abnormalities, genetic conditions, traumatic or infectious disorders, or hypoxic-ischemic insult are some of the causes of CP $[3,4]$. The patients experience loss of oligodendrocytes and myelin in the brain [5]. The distribution of the lesion in the brain gives the clinical picture of $\mathrm{CP}$. There are no specific diagnostic tests for CP. Magnetic resonance imaging (MRI), positron emission tomography [6] and single photon emission computed tomography (SPECT) scan are some of the imaging modalities for CP [4], but among all SPECT scan is more sensitive in detecting abnormalities in the brain $[7,8]$.
SPECT scan is a functional imagining modality that can provide valuable information for the diagnosis of CP [4]. SPECT scan is an effective tool for the detection of brain hypoperfusion, cerebral blood flow, cerebral impairment and local brain metabolism [9-11]. According to Lee et al. brain SPECT scan is helpful in diagnosis of CP and can detect abnormalities in cortical, sub cortical nuclei and cerebellar regions. Okumura et al. suggested that SPECT could also be useful for the diagnosis of kernicterus in CP patients [12].

SPECT using N-isopropyl-p-[123I]-iodoamphetamine is a useful tool for neurological assessment in CP patients [13]. SPECT scan can indicate improvement before the clinical changes are manifested and detect changes at the molecular level. The extent of perfusion is reflected with extent of tracer uptake. A reduced tracer uptake in these regions represents hypoperfused areas of the brain; an increased tracer uptake represents hyperperfusion while absence of tracer uptake represents the areas with no perfusion. SPECT scan reflects the extent of improvement in perfusion among the patients and regenerative potential of hESCs [9-11]. 
Citation: Shroff G, Barthakur JK, Mohan P, Mahajan H (2015) Single Photon Emission Computed Tomography Scan as a Diagnostic Tool in Children with Cerebral Palsy Treated with Human Embryonic Stem Cells. J Nucl Med Radiat Ther 6: 223. doi: $10.4172 / 2155-9619.1000223$

Page 2 of 6

Although many studies have been published related to brain perfusion abnormalities in various neurological disorders $[1,4,14]$, there is negligible literature concerning the interval changes in perfusion in the cerebral cortical functional areas, before and after the therapy.

The present study was conducted to evaluate the diagnostic modality of SPECT scan. The patients with CP were treated with human embryonic stem cell (hESC) therapy as described previously [15].

\section{Methodology}

The patients with clinical manifestations of CP underwent brain SPECT scan before and after hESC injection. The study was conducted from Oct 2007 to July 2013 in Nutech Mediworld, New Delhi, India. The study design and the detailed techniques have been elaborated in our previous study $[15,16]$. Briefly, the study consisted of four treatment phases $(\mathrm{T} 1, \mathrm{~T} 2, \mathrm{~T} 3, \mathrm{~T} 4)$ separated by a gap phase of $4-6$ months. After CP diagnosis, the patients were tested with $0.25 \mathrm{~mL}$ hESC injected subcutaneously for any hypersensitivity reactions.

During first treatment phase (8 weeks) $0.25 \mathrm{~mL}$ dose containing $<4$ million hESCs were administered through intramuscular (i.m) (for priming) route once daily and $1 \mathrm{~mL}$ dose containing of $<16$ million cells hESC was administered twice every 7 days through intravenous (iv) route (to facilitate faster transplantation and migration of the hESCs to the affected area).

Each patient also received a dose of hESCs by caudal route repeated every 5-14 days to ensure they reach the spinal fluid and regenerate the spinal cord and allow deep muscles to repair.

The patients entered second and third treatment phase (T2 and T3) after a gap period of 3-6 months, where the same dosage regime was administered. Both T2 and T3 lasted for 4 weeks and were separated by a gap phase of 3-6 months. After a gap period of 6-12 months T4 was performed with the increased IV dose of hESC by $1 \mathrm{~mL}$.

The efficacy of hESC therapy was assessed by evaluating the change in SPECT scan at the end of each treatment phase.

Nutech Mediworld is a GCP (good clinical practice) compliant certified center. The treatment carried out at the centre is approved by the Independent Ethics Committee (IEC). The Institutional Committee of Stem Cell Research and Therapy (IC-SCRT) reports all the work with respect to embryonic stem cells to National Apex Body [16].

The cell lines cultured and maintained as per our proprietary inhouse patented technology in a GMP (good manufacturing practice), GLP (good laboratory practice) and GTP (good tissue practice) certified Nutech Mediworld laboratory (Patent-WO 2007/141657A PCT/1B 2007 Published 13 December 2007). The procedures followed were in accordance with the ethical standards laid down and in conformance with the Helsinki Declaration of 1975, as revised in 2008 $[15,17]$.

\section{SPECT scan}

\section{Preparation of 99mTc-HMPAO}

Technetium-hexa methyl propylene aminoxime (Tc-HMPAO) SPECT scan was analyzed to assess the number of hypoperfused lesions/areas in the brain and the percentage of improvement by hESC treatment. A HMPAO kit was purchased from Ceretec ${ }^{\mathrm{Tn}}$, Amersham Ltd., Amersham, UK.

Fresh elutes of technetium $(99 \mathrm{mTc})$ were used to prepare $99 \mathrm{mTc}$ HMPAO according to the manufacturer's instructions and recommendations. In brief, $1110-2960 \mathrm{MBq}$ of $99 \mathrm{mTcO} 4$ in $5 \mathrm{~mL}$ of saline were added to a freeze dried Exametazime kit $\left(\right.$ Cerete $\left.^{\mathrm{Tm}}\right)$ to produce $99 \mathrm{mTc}$-HMPAO. Tc HMPAO was eluted everyday by IBA generator in the nuclear medicine department (Fortis Hospital, Vasant kunj, Delhi).

\section{SPECT acquisition}

Brain SPECT scan was performed 20 minutes after the intravenous administration of $99 \mathrm{mTc}$-HMPAO into antecubital vein of the patients in a quite dimly lit room. A tomographic camera (Millinieum MG, GE) equipped with a low-energy high resolution parallel hole collimator was used. After 15-120 minutes, the patient was placed in a supine position in order to ensure that orbito-meatal line (OML) is positioned vertically, centered in the field of view.

The SPECT scan (Millennium MG, GE) was performed before or within 7 to 10 days of hESC therapy initiation and thereafter at the end of the treatment.

\section{Gamma camera imaging}

Each scan was performed using a dual headed gamma $(\gamma)$ camera of $8.5 \mathrm{~mm}$ crystal thickness equipped with a dual Digital Correlated Signal Enhancement (CSE) detector and $55 \mathrm{ke}-540 \mathrm{keV}$ energy window connected to a computer.

Images were acquired in a step-and-shoot mode (128 projections, $35 \mathrm{sec} /$ projection) on a $128 \times 128$ matrix and the photo peak was centered at $140 \mathrm{keV}$ with a symmetrical $10 \%$ window. Images were reconstructed using the filtered back projection technique. No attenuation correction was applied.

\section{SPECT scan analysis}

We applied the NeuroGam ${ }^{\mathrm{TM}}$ software on the reconstructed data, for the evaluation of brain perfusion in specific areas in the left and right hemispheres. NeuroGam ${ }^{\mathrm{TM}}$ is a comprehensive package for the display and quantification of functional brain studies, allowing differential analysis of activation studies by using an automatic registration algorithm.

This software applies an affine co-registration by blocks of data defined in the Talairach space. Each hemisphere is subdivided in six rectangular volumes which undergo a specific affine transformation to fit the Talairach atlas. There is no additional smoothing performed. The method allows quantitative and qualitative comparison of sequential brain images on the same patient and a given patient (either with a normal database or disease specific database), creation of normal and disease specific database and, the quantitative analysis of brain images according to predefined Broadmann functional areas (Brodmann areas 1,2,3-primary somatosensory cortex; area 4-primary motor cortex, area 17-primary visual cortex and areas 41 and 42primary auditory cortex). 
Citation: Shroff G, Barthakur JK, Mohan P, Mahajan H (2015) Single Photon Emission Computed Tomography Scan as a Diagnostic Tool in Children with Cerebral Palsy Treated with Human Embryonic Stem Cells. J Nucl Med Radiat Ther 6: 223. doi: $10.4172 / 2155-9619.1000223$

Page 3 of 6

\section{Image processing and interpretation}

SPECT scans were interpreted by a certified nuclear medicine physician with an extensive experience in brain SPECT scans interpretation. We used a previously described approach for interpreting functional imaging scans such as grey color representing normal regions in the brain; red, pink and white representing above normal regions; while green, light/dark blue, black representing hypoperfused regions [15].

Patients showing $60-90 \%$ changes were considered to have significant improvement, $30-60 \%$ changes moderate improvement and $10-30 \%$ changes mild improvement. In addition, the evaluation of the motor functions of cerebral palsy cases was done with GMFCS, which is an international parameter with grades $5 \rightarrow 1$ in $\mathrm{BAD} \rightarrow \mathrm{GOOD}$ direction.

\section{Results}

\section{Study Patients}

The data of 88 patients were analyzed, of which 24 subjects were females. The age of the patients varied from 2 months- 18 years. The total treatment days varied from 31 to 310 days. The demographic details of the patients is given in Table 1.

\begin{tabular}{|l|l|}
\hline Characteristics & N \\
\hline Gender & \\
\hline Male & 23 \\
\hline Female & 64 \\
\hline Origin & \\
\hline India & 81 \\
\hline Abroad & 7 \\
\hline Age & \\
\hline Up to 2 years & 11 \\
\hline $2-5$ years & 27 \\
\hline $5-10$ years & 25 \\
\hline $10-20$ years & 25 \\
\hline N: No. of patients & \\
\hline
\end{tabular}

Table 1: Demographic details of the patients.

\section{Outcome}

All the subjects showed an improvement in SPECT scan. Majority of subjects $(n=58)$ had significant SPECT scan $(>60 \%)$ change, followed by moderate SPECT scan $(>30 \%<60 \%)$ change in 15 subjects, and mild scan $(>10 \%<30 \%)$ change in three subjects (Figure $1)$.

None of the subjects showed deterioration. The SPECT scan report of one patient is illustrated in Figures $2 \mathrm{~A}$ and $2 \mathrm{~B}$.

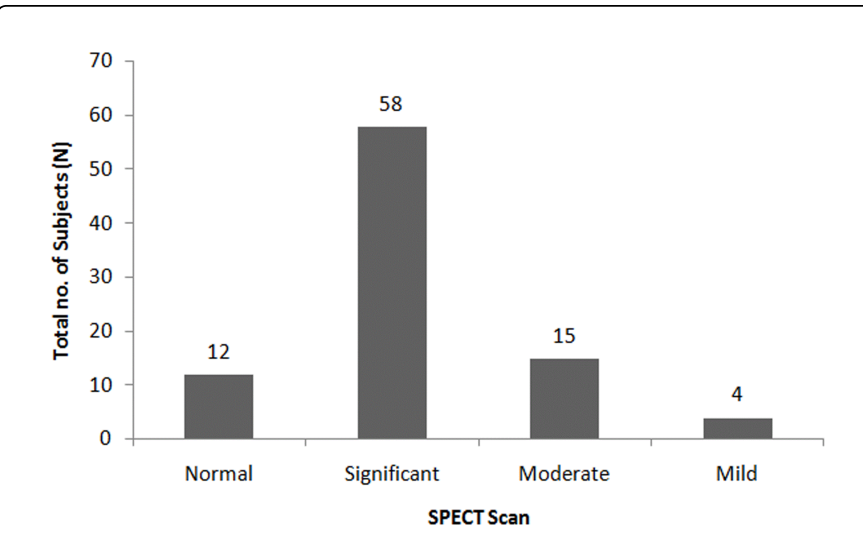

Figure 1: Change in SPECT scan at the end of the treatment.

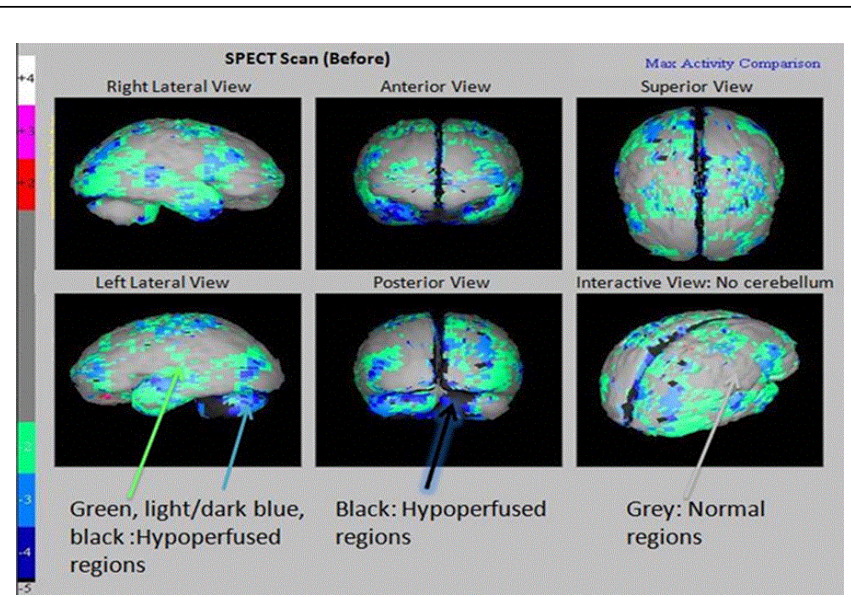

Figure 2A: SPECT Scan of Cerebral Palsy Patient Showing Hypoperfused Regions before receiving hESCs Therapy.

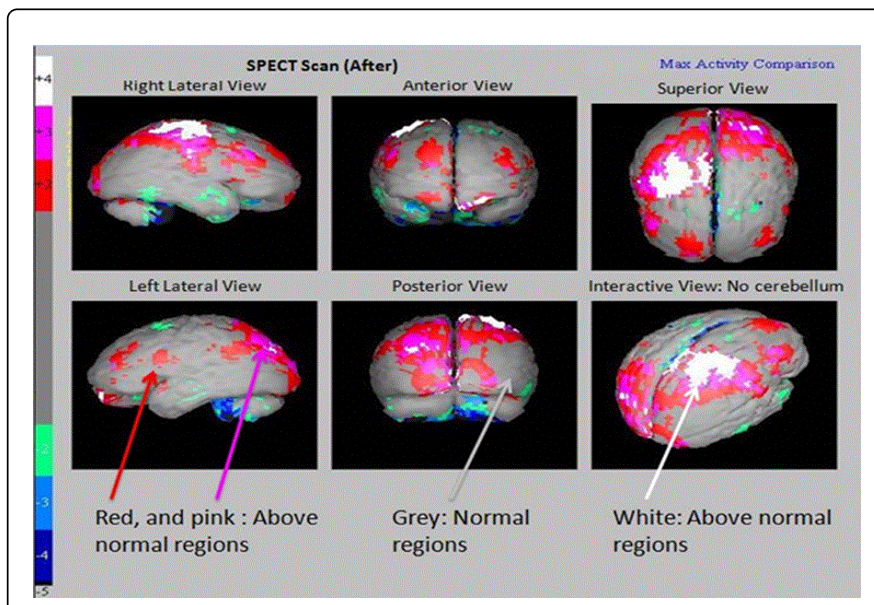

Figure 2B: SPECT Scan of a Cerebral Palsy Patient Showing Improved Perfusion (Reduced Black Areas) after receiving hESCs Therapy. 
Citation: Shroff G, Barthakur JK, Mohan P, Mahajan H (2015) Single Photon Emission Computed Tomography Scan as a Diagnostic Tool in Children with Cerebral Palsy Treated with Human Embryonic Stem Cells. J Nucl Med Radiat Ther 6: 223. doi: $10.4172 / 2155-9619.1000223$

Page 4 of 6

All the patients were assessed clinically using GMFCS scores before and after the therapy. The improvement in SPECT scan after the therapy was also reflected in the improvement in GMFCS scores of the patients after the therapy. GMFCS Score of the patients at start and end of each treatment phase Table 2.

\begin{tabular}{|l|l|l|l|l|l|}
\hline & 1 & 2 & 3 & 4 & $\mathbf{5}$ \\
\hline & \multicolumn{4}{|l|}{ GMFCS SCORE } \\
\hline Baseline (N=88) & 1 & 12 & 14 & 21 & 40 \\
\hline End of T1 & 6 & 31 & 36 & 14 & 1 \\
\hline $\begin{array}{l}\text { Start of T2 } \\
\text { (N=65) }\end{array}$ & 1 & 22 & 29 & 13 & 0 \\
\hline End of T2 & 7 & 34 & 17 & 7 & 0 \\
\hline $\begin{array}{l}\text { Start of T3 } \\
\text { (N=38) }\end{array}$ & 3 & 17 & 11 & 7 & 0 \\
\hline End of T3 & 11 & 18 & 6 & 3 & 0 \\
\hline $\begin{array}{l}\text { Start of T4 } \\
\text { (N=15) }\end{array}$ & 1 & 8 & 6 & 0 & 0 \\
\hline End of T4 & 5 & 7 & 3 & 0 & 0 \\
\hline
\end{tabular}

Table 2: GMFCS Score of the Patients at Start and End of Each Treatment Phase.

\section{Discussion}

In the present study the efficacy of hESC therapy was evaluated based on the grade in SPECT scan and change in GMFCS scores. An improvement in SPECT scan was observed in CP patients after hESCs therapy. The improvement was reflected by a reduced hypoperfusion in the SPECT scan of the brain. A significant change in SPECT scan $(>60 \%)$ was observed in majority of subjects. This improvement was also reflected in GMFCS scores change, where GMFCS score was reduced from 5 (BAD) to 1 (GOOD) in majority of the patients at the end of the treatment. These results indicate that SPECT scan is a useful tool in the assessment of patients with $\mathrm{CP}$ and treatment progression with hESCs.

SPECT is a physiological imaging technique that detects metabolic and neurochemical changes in neurogenerative disorder [14]. SPECT is an aid that may help in diagnosing the disease at an early stage. According to Wang et al. combination of SPECT with other techniques such as olfaction test and transcranial sonography may result in higher accuracy of neurogenerative disease diagnosis [14]. In our study, the CP patients were evaluated for recovery using SPECT scan. A reduced tracer uptake in the hypoperfused regions of the brain can be detected by the SPECT scan. hESCs migrated to the affected area and the improvement in perfusion is evident from the extent of improvement in the SPECT scan. At the end of therapy 65.9\% $(\mathrm{N}=58)$ subjects showed $>60 \%$ of improvement in SPECT scan. This improvement was reflected clinically in the GMFCS scores.

In the present study, SPECT imaging was performed after injection of radiotracer, TC-99m HMPAO. The radiotracer, 99mTc labeled HMPAO SPECT is the most widely used radiotracers for imaging cerebral blood flow [18]. This radiotracer is small, lipophilic and stable that circulates to brain with the blood, readily passes through the BBB and is metabolized and retained intracellularly. The brain distribution of HMPAO reflects regional brain perfusion [19,20]. A normal SPECT scan with 99mTc-HMPAO shows more intense perfusion in the cerebellum [21]. 99mTc-HMPAO is more preferred radiotracer than $99 \mathrm{mTc}$ ethyl cysteinate dimer (ECD) [7].

In relation to clinical outcomes, SPECT scans have false positive and false negative rate, hence more studies to measure the accuracy of a diagnostic test are needed [22]. Also, diagnostic accuracy statistics of SPECT should also be examined for insight into the common rates of false positive and negative tests [23]. Valkama et al. in their study reported $100 \%$ sensitivity and $67 \%$ specificity of SPECT scan in predicting moderate or severe $\mathrm{CP}$. The perfusion defects in $\mathrm{CP}$ were predicted with sensitivity of $80 \%$, specificity of $70 \%$ and accuracy of $74 \%$ [24]. A high diagnostic sensitivity of SPECT scan shows that costly false-negative SPECT scans are rare [23].

Neuroimaging studies have increased our understanding of the brain abnormalities in the patients with CP [25]. SPECT brain perfusion imaging is more valuable and sensitive than $\mathrm{X}$ ray, CT or MRI as it can detect more brain lesions compared to other morphologic modalities [7,8]. MRI detects only damage that occurred when flow of cerebral blood is reduced to $<10 \mathrm{ml} / 100 \mathrm{~g}$ of tissue/1 min, while SPECT scan have ability to detect hypoperfusion areas secondary to neuronal loss [24]. MRI and CT are less informative due to common problems associated with them such as inappropriate and inconsistent morphologic, radiologic and, pathologic findings and their uncertain relationship to the time estimation of brain injury [25].

Abdel Dayem et al. in their study reviewed the data of 228 patients of age 11-88 years with traumatic brain injury. SPECT scan revealed focal area hypoperfusion in $77 \%$ of the patients. Of 41 patients who had normal CT and negative loss of consciousness, 28 patients were reported to have abnormal brain SPECT scan [7]. Abu Judeh et al. observed brain perfusion abnormalities detected by SPECT scan in patients who previously had normal X ray CT for brain lesions. The incidence of abnormal brain perfusion was greater in the patients who were imaged early at $<3$ months post injury than those imaged delayed at $>3$ months from date of accident. Hence, SPECT scan must be performed at an early stage [8]. In a study by Lee et al., cerebellar hypoperfusion was reported in $20(\mathrm{~N}-52) \mathrm{CP}$ patients as demonstrated by SPECT scan, while MRI detected cerebellar atrophy only in one patient [4]. Ryu et al. compared SPECT scan and MRI findings in assessments of abnormalities in patients with autism. SPECT scan and MRI were performed for 23 patients, of which 20 patients had abnormal SPECT scan revealing decreased perfusion in the cerebellar regions, while all patients had normal MRI without evidence of cerebellar abnormalities. Hence, SPECT scan is more sensitive in detecting abnormalities than MRI [23].

Stem cells follow a chain of reactions to replace the damaged neurons involving both exogenous and endogenous neurons, glial cells, and endothelial cells. These cells act synergistically to repair brain damage [26]. Various studies demonstrated the differentiation of hESCs derived progenitor cells to the site of brain injury [27-29], that raised the hope that hESC therapy can be developed to treat central nervous system related disorders [30]. In our previous study, we have observed successful treatment of the CP patients with hESCs therapy [15]. It is possible that the hESCS injected in our patients, could have permeated parenchyma via blood brain barrier (BBB) and migrated to the affected regions, as the size of the hESCs were $<1 \mu \mathrm{m}$. These cells might have differentiated into neural cells and replaced the damaged tissue [15]. In another paper the co-relation between SPECT scan and 
Citation: Shroff G, Barthakur JK, Mohan P, Mahajan H (2015) Single Photon Emission Computed Tomography Scan as a Diagnostic Tool in Children with Cerebral Palsy Treated with Human Embryonic Stem Cells. J Nucl Med Radiat Ther 6: 223. doi: $10.4172 / 2155-9619.1000223$

Page 5 of 6

GMFCS has been done. We have observed a lot of cognitive improvement that was not determined by GMFCS $[15,16]$.

In the present study, etiology of $\mathrm{CP}$ was hypoxia prenatally, especially at delivery (traumatic). hESCs were administered through i.m, i.v and caudal routes. We observed that our patients showed an improvement in SPECT scan earlier than the clinical improvement. This might be due to the fact that hESCs need time for regeneration and differentiation and become functional. This was also the primary reason for gap phase of 3-6 months between the successive treatment phases in our hESC therapy protocol. Our results suggest that the application of NeuroGam processing protocol offers a more detailed and objective evaluation of regional cerebral blood flow and may contribute to a better discrimination in monitoring any treatment response in patients. The age of the patients was between 2 months- 18 years and total treatment days varied from 31 to 310 days. In such a short duration of time, it is highly unlikely that the cells of the brain matured and healed naturally, hence we could assume that that improvement in motor function was due to stem cell therapy. None of our patient had reported deterioration and their condition was stable.

The results of the present study were derived from a small cohort of patients, and need to be replicated in a larger sample population. Moreover, the comparison of brain perfusion with clinical and neuropsychological parameters in neurological patients needs further investigation.

\section{Conclusion}

The extent of improvement in patients treated with hESCS was carefully monitored with SPECT scan and GMFCS scores. Brain SPECT scan may be extremely useful tools in monitoring the treatment response to hESCs therapy in patients of CP. Further studies must be conducted to examine the diagnostic value SPECT scans and to evaluate the possible effects of hESC therapy. SPECT imaging helps in understanding the differential diagnosis and the disease progression. SPECT scan may be used as a prognostic tool for the detection of the extent of improvement in perfusion in the brain. SPECT scan can be used as an effective diagnostic modality to assess the patient with $\mathrm{CP}$ before and after hESCS therapy.

\section{Conflict of Interest}

The authors do not have any conflict of interest.

\section{Acknowledgements}

The authors acknowledge Dr. Anupama Gupta (Pediatrician, Max Hospital, Saket, New Delhi), the staff of Nutech Mediworld, all patients and their parents. The authors also acknowledge Knowledge Isotopes Pvt. Ltd (http://www.knowledgeisotopes.com) for writing support.

\section{References}

1. Chang CP, Shiau YC, Wang JJ, Ho ST, Kao A (2002) Abnormal regional cerebral blood flow on $99 \mathrm{mTc}$ ECD brain SPECT in patients with primary Sjogren's syndrome and normal findings on brain magnetic resonance imaging. Ann Rheum Dis 61: 774-778.

2. Roll S, Müller-Nordhorn J, Keil T, Scholz H, Eidt D, et al. (2008) Dacron vs. PTFE as bypass materials in peripheral vascular surgery--systematic review and meta-analysis. BMC Surg 8: 22
3. Carroll JE, Mays RW (2011) Update on stem cell therapy for cerebral palsy. Expert Opin Biol Ther 11: 463-471.

4. Lee JD, Kim DI, Ryu YH, Whang GJ, Park CI, et al. (1998) Technetium-99m-ECD brain SPECT in cerebral palsy: comparison with MRI. J Nucl Med 39: 619-623.

5. Goldman SA, Schanz S, Windrem MS (2008) Stem cell-based strategies for treating pediatric disorders of myelin. Hum Mol Genet 17: R76-83.

6. Inzucchi SE, Bergenstal RM, Buse JB, Diamant M, Ferrannini E, et al. Management of hyperglycemia in type 2 diabetes: a patient-centered approach: position statement of the American Diabetes Association (ADA) and the European Association for the Study of Diabetes (EASD). Diabetes Care 35: 1364-1379.

7. Abdel-Dayem HM, Abu-Judeh H, Kumar M, Atay S, Naddaf S, et al. (1998) SPECT brain perfusion abnormalities in mild or moderate traumatic brain injury. Clin Nucl Med 23: 309-317.

8. Abu-Judeh HH, Parker R, Singh M, el-Zeftawy H, Atay S, et al. (1999) SPET brain perfusion imaging in mild traumatic brain injury without loss of consciousness and normal computed tomography. Nucl Med Commun 20: 505-510.

9. Dierckx RA, Vandevivere J, Dom L, Melis K, Janssens G, et al. (1992) Single photon emission computed tomography using perfusion tracers in seizure disorders. Epilepsy Res 12: 131-139.

10. Mohan P, Mahajan H, Shroff G (2014) Serial brain SPECT imaging in monitoring treatment response to stem cell therapy in cerebral palsy patients. J Nucl Med 55: 1850.

11. Catafau AM1 (2001) Brain SPECT in clinical practice. Part I: perfusion. J Nucl Med 42: 259-271.

12. Okumura A, Hayakawa F, Maruyama K, Kubota T, Kato K, et al. (2006) Single photon emission computed tomography and serial MRI in preterm infants with kernicterus. Brain Dev 28: 348-352.

13. Yamada K, Tsuzura S, Matsuda H (1995) Brain MRI and single photon emission computed tomography in severe athetotic cerebral palsy: a comparative study with mental and motor disorders. No To Hattatsu 27: 269-275.

14. Wang L, Zhang Q, Li H, Zhang H (2012) SPECT molecular imaging in Parkinson's disease. J Biomed Biotechnol 2012: 412486.

15. Shroff G, Gupta A, Barthakur J (2014) Therapeutic potential of human embryonic stem cell transplantation in patients with cerebral palsy. J Transl Med 12: 318 .

16. Shroff G, Das L (2014) Human Embryonic Stem Cell Therapy in Cerebral Palsy Children with Cortical Visual Impairment: A Case Series of 40 Patients. Journal of Cell Science \& Therapy 5: 51-57.

17. World Medical Association (2002) World Medical Association: Declaration of Helsinki: ethical principles for medical research involving human subjects. J Postgrad Med 48: 206-208.

18. Neirinckx RD, Canning LR, Piper IM, Nowotnik DP, Pickett RD, et al. (1987) Technetium-99m d,l-HM-PAO: a new radiopharmaceutical for SPECT imaging of regional cerebral blood perfusion. J Nucl Med 28: 191-202.

19. Ogasawara K, Ogawa A, Ezura M, Konno H, Suzuki M, et al. (2001) Brain single-photon emission CT studies using 99mTc-HMPAO and 99mTcECD early after recanalization by local intraarterial thrombolysis in patients with acute embolic middle cerebral artery occlusion. AJNR Am J Neuroradiol 22: 48-53.

20. Lassen NA, Andersen AR, Friberg L, Paulson OB (1988) The retention of [99mTc]-d,l-HM-PAO in the human brain after intracarotid bolus injection: a kinetic analysis. J Cereb Blood Flow Metab 8: S13-S22.

21. Catafau AM, Lomeña FJ, Pavia J, Parellada E, Bernardo M, et al. (1996) Regional cerebral blood flow pattern in normal young and aged volunteers: a 99mTc-HMPAO SPET study. Eur J Nucl Med 23: 1329-1337.

22. Braile MC, Carnevalli NC, Goissis G, Ramirez VA, Braile DM (2011) In vitro properties and performance of glutaraldehyde-crosslinked bovine pericardial bioprostheses treated with glutamic acid. Artif Organs 35: 497-501. 
Citation: Shroff G, Barthakur JK, Mohan P, Mahajan H (2015) Single Photon Emission Computed Tomography Scan as a Diagnostic Tool in Children with Cerebral Palsy Treated with Human Embryonic Stem Cells. J Nucl Med Radiat Ther 6: 223. doi: $10.4172 / 2155-9619.1000223$

Page 6 of 6

23. Ryu YH, Lee JD, Yoon PH, Kim DI, Lee HB, et al. (1999) Perfusion impairments in infantile autism on technetium-99m ethyl cysteinate dimer brain single-photon emission tomography: comparison with findings on magnetic resonance imaging. Eur J Nucl Med 26: 253-259.

24. Valkama AM, Ahonen A, Vainionpaa L, Torniainen P, Lanning P, et al. (2001) Brain single photon emission computed tomography at term age for predicting cerebral palsy after preterm birth. Biol Neonate 79: 27-33.

25. Korzeniewski SJ, Birbeck G, DeLano MC, Potchen MJ, Paneth N (2008) A systematic review of neuroimaging for cerebral palsy. J Child Neurol 23: 216-227.

26. Pabon MM, Borlongan CV (2013) Advances in the Cell-Based Treatment of Neonatal Hypoxic-Ischemic Brain Injury. Future Neurol 8: 193-203.

27. Liu YP, Seçkin H, Izci Y, Du ZW, Yan YP, et al. (2009) Neuroprotective effects of mesenchymal stem cells derived from human embryonic stem cells in transient focal cerebral ischemia in rats. J Cereb Blood Flow Metab 29: 780-791.

28. Ma J, Wang Y, Yang J, Yang M, Chang KA, et al. (2007) Treatment of hypoxic-ischemic encephalopathy in mouse by transplantation of embryonic stem cell-derived cells. Neurochem Int 51: 57-65.

29. Yang D, Zhang ZJ, Oldenburg M, Ayala M, Zhang SC (2008) Human embryonic stem cell-derived dopaminergic neurons reverse functional deficit in parkinsonian rats. Stem Cells 26: 55-63.

30. Filipovic R, Santhosh Kumar S, Fiondella C, Loturco J (2012) Increasing doublecortin expression promotes migration of human embryonic stem cell-derived neurons. Stem Cells 30: 1852-1862. 